\title{
On the Analysis of Average Time Complexity of Estimation of Distribution Algorithms
}

\author{
Tianshi Chen, Ke Tang, Guoliang Chen and Xin Yao
}

\begin{abstract}
Estimation of Distribution Algorithm (EDA) is a well-known stochastic optimization technique. The average time complexity is a crucial criterion that measures the performance of the stochastic algorithms. In the past few years, various kinds of EDAs have been proposed, but the related theoretical study on the time complexity of these algorithms is relatively few. This paper analyzed the time complexity of two early versions of EDA, the Univariate Marginal Distribution Algorithm (UMDA) and the Incremental UMDA (IUMDA). We generalize the concept of convergence to convergence time, and manage to estimate the upper bound of the mean First Hitting Times (FHTs) of UMDA (IUMDA) on a well-known pseudo-modular function, which is frequently studied in the field of genetic algorithms. Our analysis shows that UMDA (IUMDA) has $O(n)$ behaviors on the pseudo-modular function. In addition, we analyze the mean FHT of IUMDA on a hard problem. Our result shows that IUMDA may spend exponential generations to find the global optimum. This is the first time that the mean first hitting times of UMDA (IUMDA) are theoretically studied.
\end{abstract}

\section{INTRODUCTION}

Belonging to the family of Evolutionary Algorithms (EAs), Estimation of Distribution Algorithms (EDAs) [1] are a new kind of population-based stochastic algorithms for optimization. In each generation, EDAs create a new population of solutions and select some of them for estimating the joint probability of solutions. The general procedure of EDA is shown in Table 1. In the past years, many variants of EDAs have been proposed, but the theoretical study related to the time complexity of these algorithms is relatively few. In [2], Mühlenbein and Schlierkamp-Voosen studied the time to convergence of constant selection intensity algorithms on OneMaX function. Later, Mühlenbein [3] studied the response to selection (RS) equation of the Univariate Marginal Distribution Algorithm (UMDA) [3] on ONEMAX function through experiments and theoretical analysis. Pelikan et al. [4] studied the time to convergence of BOA on ONEMAX function. Droste [5] gave rigorous analysis of the runtime of compact genetic algorithm (cGA) [6] on linear functions. Rastegar and Meybodi [7] carried out theoretical study on the global convergence time of a limit model of EDAs by drift analysis, but they did not reveal any relations between the problem size and computation time of EDAs. González et al. [8] studied the average First Hitting Time (FHT)

The authors are with the Nature Inspired Computation and Applications Laboratory (NICAL), the University of Science and Technology of China, Hefei 230027, Anhui, China. Xin Yao is also with the Centre of Excellence for Research in Computational Intelligence and Applications (CERCIA), the School of Computer Science, the University of Birmingham, Edgbaston, Birmingham B15 2TT, UK. (Email: cetacy@mail.ustc.edu.cn, ketang@ustc.edu.cn, glchen@ustc.edu.cn, x.yao@cs.bham.ac.uk. WWW: http://nical.ustc.edu.cn) of three different types of EDAs, including UMDA, on LINEAR function, LEADINGONES function [9], [10], [11] and UNIMAX (long-path) function [12], but these results were observed from some experiments, no theoretical result had been given.

The FHT is defined as the first time for a stochastic optimization algorithm to reach the global optimum, and it is often used to measure the time complexity of GAs [10], [14], [15]. Since EDAs are also stochastic algorithms, their time complexity can also be measured by the FHT. Recent works pointed out the significance of studying the FHT of EDAs [16], [17]. But the related theoretical work is few.

In this paper, we aim at analyzing the mean FHTs of two early versions of EDA, the UMDA and the Incremental UMDA (IUMDA) [3], with two different selection schemes. Our analysis is mainly carried out on a simple pseudomodular function, which is frequently studied in the field of the time complexity of GAs [10], [11], called LEADINGONES function [9]. The function is $f(\mathbf{x})=\sum_{i=1}^{n} \prod_{j=1}^{i} x_{j}$, where $x_{j}=0$ or $1\left(\left(x_{1}, x_{2}, \ldots, x_{n}\right)\right.$ describes a individual $)$. As we stated above, UMDA's behavior on this function has been experimentally studied in [8]. The experiment shows that the mean FHT of UMDA on LEADINGONES function performs an $O(n)$ behavior, but this observation is not supported by any theoretical result. In this paper, we give rigorous analysis for UMDA and IUMDA with different selection schemes on this pseudo-modular function. In addition, we find a problem that takes the IUMDA at least exponential generations on average, to find its global optimum, theoretical analysis is carried out.

The rest of the paper is organized as follows: Section 2 gives the preliminaries; Section 3 discusses the convergence times of IUMDA and UMDA with truncation selection, so as to bound the mean FHTs; Section 4 analyzes the UMDA with 2 -tournament selection; Section 5 discusses an exponential case of the IUMDA; Section 6 concludes the paper.

\section{Preliminaries}

\section{A. First Hitting Time and Convergence Time}

The formal definition of FHT, denote by $\tau$, is

$$
\tau=\min \left\{t: \mathbf{x}^{*} \in \xi_{t}\right\}
$$

where $\xi_{t}$ is the population in the $t$-th generation of an EA, and $\mathbf{x}^{*}$ is the global optimum of a given problem. He and Yao proposed two techniques, drift analysis [10] and analytical Markov chain [14], to estimate the mean FHT of Genetic Algorithms (GAs). 
TABLE I

THE GENERAL PROCEDURE OF EDA

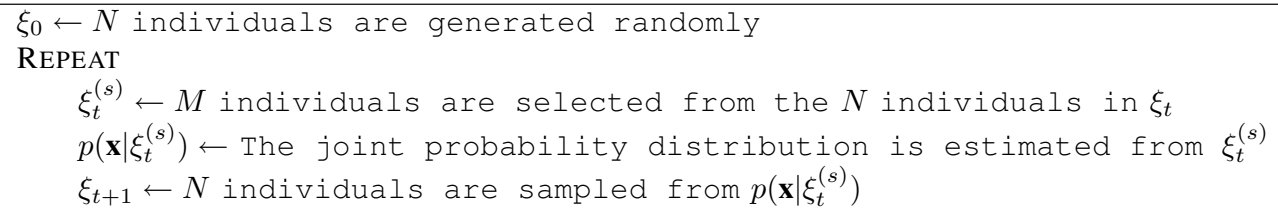

Another concept "convergence" is often used to measure the limit behavior of EAs, which was derived from the concept of convergence of random sequences [9]. It describes the situation that all the individuals of a population reach the global optimum. Thus "An EA (on a problem) converges to the global optimum" implies "The EA (on the problem) has found the global optimum", but "An EA (on a problem) has found the global optimum" doesn't always imply that "The EA (on the problem) will converge to the global optimum".

Convergence describes the limit behaviors of EAs, not the time complexity of EAs. But if we assume that an EA on a problem will converge to the global optimum in finite time, we can then measure the time complexity of EAs by recording the minimal number of generations for an EA to converge. This number is called Convergence Time (CT), denoted by $T$. FHT and CT are actually closely related to each other. The FHT describes the situation that one of the individuals in the population reach the global optimum for the first time, and the CT describes the situation that all individuals in the population reach the global optimum. In most cases, CT is longer than FHT (for $(1+1)$ EAs, they are equivalent), so we can further record the number of generations between "first hitting" and "convergence", called Converging Time (CingT), denoted by $C$, and for an EA,

$$
T=\tau+C, \quad C \geq 0 .
$$

\section{B. Measure the Time Complexity of EDAs}

In this subsection, we will give the corresponding formal definition of the concepts mentioned in the previous subsection for EDAs. The definition of FHT is the same as (1). The formal definition of "convergence" was given by Zhang and Mühlenbein [18], as follows,

$$
\lim _{t \rightarrow \infty} \bar{F}(t)=G^{*},
$$

where $\bar{F}(t)$ is the average fitness of individuals in the $t$-th generation, $G^{*}$ is the fitness of global optimum. In [18], Zhang and Mühlenbein also studied the convergence properties of EDAs under the assumption

$$
p\left(\mathbf{x} \mid \xi_{t+1}\right)=p\left(\mathbf{x} \mid \xi_{t}^{(s)}\right),
$$

It was shown that EDAs with proportion selection, truncation selection and tournament selection will all converge to the global optimum under the above assumption. Under a very big population size and the assumption of (3), we further define the CT for an EDA as

$$
T=\min \left\{t: p\left(\mathbf{x}^{*} \mid \xi_{t}^{(s)}\right)=1\right\},
$$

where $\mathbf{x}^{*}$ is the global optimum of a given problem, $p\left(\mathbf{x}^{*} \mid \xi_{t}^{(s)}\right)$ is the estimated distribution of an EDA in the $t$-th generation. This definition is valid if we only care about the time complexity of EDAs instead of the convergence property of EDAs. In ther words, we always assume that the EDAs will converge to the global optimum to obtain the time complexity results on EDAs in the following parts of the paper.

The mean CT is given by $\mathbb{E}[T]$ and the mean FHT is $\mathbb{E}[\tau]$. According to (2),

$$
\mathbb{E}[\tau] \leq \mathbb{E}[T]
$$

(5) shows us a way for estimating the upper bound of the mean FHT by estimating the upper bound of the mean CT.

\section{Domino Convergence}

Rudnick [19] introduced a maximization problem which has an exponential scaled fitness structure, called BININT problem, defined as:

$$
\operatorname{BinInT}(x)=\sum_{i=1}^{n} x_{i} 2^{n-i}, \quad x_{i} \in\{0,1\},
$$

where $n$ is the length of the binary string. Let us consider a selection process with crossover (e.g., a pure truncation selection process with uniform crossover), on BININT problem. Initialized with a population of random individuals, it is easy for us to find that the bits of all the individuals will finally converge to 1 one after another. Rudnick called this sequential convergence phenomenon Domino convergence. Thierens et al. [20] study the convergence time of this process. It was shown that $O(n)$ generations are enough when the selection intensity is constant. In fact, the domino convergence also occur in the case of LEADINGONES problem. The fitness of an individual $\mathbf{x}$ is determined by the number of the leading 1-bits of $\mathbf{x}$, and it is not influenced by any bits right to the leftmost 0-bit of $\mathbf{x}$. In other words, only the leading onebits will be exposed to the selection pressure, the value of the other bits will not be influenced by selection operators. Thus, we can imagine that the domino convergence may also occur on it, and we can use this property in the analysis of some simple EDAs to get the upper bound of the convergence time of the EDAs. 


\section{UMDA and IUMDA}

The Univariate Marginal Distribution Algorithm (UMDA) is a discrete EDA [3]. As one of the earliest version of EDA, it doesn't consider the dependencies of variables. A more general version of UMDA is the Incremental UMDA [3] (or Simple UMDA [1]), IUMDA incorporates a control parameter $0<\lambda \leq 1$ on the update of the estimated probability distribution (when $\lambda=1$, IUMDA = UMDA).

The general procedure of IUMDA is shown in Table 2, where $p_{t, i}\left(x_{i}\right)$ is the estimated probability of the $i$-th bit of a individual to be $x_{i}$ in the $t$-th generation, $r_{t, i}\left(x_{i}\right)$ is the marginal frequency of the $i$-th bit of individuals to be $x_{i}$ in the $t$-th generation,

$$
r_{t, i}\left(x_{i}\right)=\frac{1}{N} \sum_{\mathbf{x} \in \xi_{t}} \delta\left(\mathbf{x} \mid x_{i}\right) .
$$

where all individuals in $\xi_{t}$ are sampled by the joint probability distribution

$$
p\left(\mathbf{x} \mid \xi_{t-1}^{(s)}\right)=\prod_{i=1}^{n} p_{t-1, i}\left(x_{i}\right),
$$

and $\delta\left(\mathbf{x} \mid x_{i}\right)$ is defined by,

$$
\delta\left(\mathbf{x} \mid x_{i}\right)= \begin{cases}1, & \mathbf{x}_{i}=x_{i} \\ 0, & \mathbf{x}_{i} \neq x_{i} .\end{cases}
$$

In the following parts of the paper, we will use $\overline{\mathbb{E}}[T]$ to bound the mean first hitting time of IUMDA and UMDA. It is noteworthy that all the analysis below is based on the following assumptions:

Assumption 1:

$$
\forall t>0,0 \leq i \leq n: \quad r_{t, i}\left(x_{i}\right)=p_{t-1, i}\left(x_{i}\right) .
$$

The above assumption is also used in [18]. In other words, we always assume that IUMDA maintains a very big population.

In addition, the evolution path of the marginal probability $p_{., i}\left(x_{i}\right)(\forall i=1, \ldots, n)$ can be considered as a random sequence $\left\{p_{t, i}\left(x_{i}\right) ; t=0,1, \ldots\right\}$, and

$$
\begin{gathered}
\bar{p}_{0, i}\left(x_{i}\right)=p_{0, i}\left(x_{i}\right), \\
\bar{p}_{t, i}\left(x_{i}\right)=\mathbb{E}\left[p_{t, i}\left(x_{i}\right) \mid \overline{\mathbf{p}}_{t-1}(\mathbf{x})\right],
\end{gathered}
$$

where $\overline{\mathbf{p}}_{t}(\mathbf{x})=\left(\bar{p}_{t, 1}\left(x_{1}\right), \bar{p}_{t, 2}\left(x_{2}\right), \ldots, \bar{p}_{t, n}\left(x_{n}\right)\right)$. In our paper, we mainly consider the sequence $\left\{\bar{p}_{t, i}\left(x_{i}\right) ; t=\right.$ $0,1, \ldots\}$, which is the expected case of the $\left\{p_{t, i}\left(x_{i}\right) ; t=\right.$ $0,1, \ldots\}$. We have the following assumption

Assumption 2:

$$
\mathbf{p}_{t}(\mathbf{x})=\overline{\mathbf{p}}_{t}(\mathbf{x}) .
$$

In the rest pasts of the paper, we will give rigorous analysis on the FHTs of UMDA or IUMDA, based on the above two assumptions.

\section{FHTS OF IUMDA AND UMDA WITH TRUNCATION SELECTION ON LEADINGONES FUNCTION}

In this section, we are trying to study the upper bound of the FHTs of IUMDA and UMDA with Truncation Selection on LEADINGONES Function. It was shown in Section 2, that
FHTs are bounded by CTs, hence we will study the CTs first. Thieren et al. [20] has utilized an approach of deriving CT by average fitness of a population and the selection intensity, but this approach is designed for a selection process. In order to analyze the IUMDA, we will calculate the cumulation of all the marginal probability distributions directly. There is a simple lemma:

Lemma 1: For IUMDA on LEADINGONES function, if at the $t_{0}$-th generation, $p_{t_{0}, i}(1)=1$, then for $\forall t>t_{0}, p_{t, i}(1)=$ 1 .

Proof: At the $t_{0}$-th generation, we have $p_{t_{0}, i}(1)=1$. According to the marginal frequencies' updating rule of IUMDA, we have,

$$
p_{t_{0}+1, i}(1)=p_{t_{0}, i}(1)+\lambda\left(r_{t_{0}, i}^{(s)}(1)-p_{t_{0}, i}(1)\right)=1 .
$$

Moreover, if $p_{k-1, i}(1)=1$, we can induce the case of the $k$-th generation:

$$
p_{k, i}(1)=p_{k-1, i}(1)+\lambda\left(r_{k-1, i}^{(s)}(1)-p_{k-1, i}(1)\right)=1 .
$$

Hence for $\forall t>t_{0}, p_{t, i}(1)=1$.

Lemma 2: For IUMDA with truncation selection, the proportions of the best individuals before and after selection in the $t$-th generation satisfy:

$$
R_{t}^{(s)}=\left\{\begin{array}{cl}
\frac{R_{t} N}{M}, & R_{t} \leq \frac{M}{N}, \\
1, & R_{t}>\frac{M}{N},
\end{array}\right.
$$

where $R_{t}$ and $R_{t}^{(s)}$ are the proportions of the best individuals before and after truncation selection.

Proof: The numbers of the best individuals before selection is $R_{t} N$. After truncation selection, all of them should be selected when $R_{t} N \leq M$; Part of them can be selected when $R_{t} N>M$, but already takes over the whole population after selection.

Now we define the $i$-convergence time $T_{i}$ to be the number of generations for a discrete EDA to converge to the global optimum on the $i$-th bit of the solution. It is defined formally as follows

$$
T_{i}=\min \left\{t: p_{t, i}\left(x_{i}^{*}\right)=1\right\},
$$

where $x_{i}^{*}$ is $i$-th locus of the global optimum.

Then we have the following theorem:

Theorem 1: For IUMDA with truncation selection on LEADINGONES function, its convergence time satisfies:

$$
\begin{aligned}
& \mathbb{E}\left[T \mid \forall t>0,0 \leq i \leq n: r_{t, i}\left(x_{i}\right)=p_{t-1, i}\left(x_{i}\right)\right] \\
&<\frac{n \ln \frac{2 M}{N}}{\ln \left(1+\frac{\lambda(N-M)}{M}\right)}+n .
\end{aligned}
$$

Proof: According to Lemma $1,{ }^{M}$ for IUMDA on LEADINGONES function, once a bit converge to 1 , it will never be 0 again. First, we can estimate $\tau_{1}$ under the average behavior of IUMDA. We assume that here the fitness of the best individuals is bigger than 1 , thus all the first bits of those individuals should be 1 , the generation begins with $t=1$. According to Lemma 2, the proportion of the best individuals after truncation selection is known, so the marginal frequency of the first bit to be 0 is known. Hence according to the 


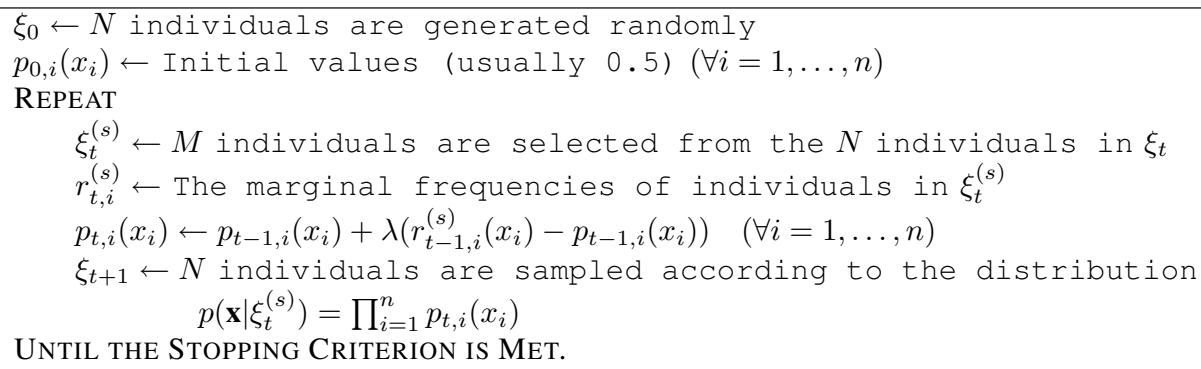

updating rule of $p_{., 1}(1)$ and Lemma 2 , when $\frac{M}{N} \geq p_{t-1,1}(0)$, we have

$$
\begin{aligned}
& \overline{\mathbb{E}}\left[p_{t, 1}(1) \mid \mathbf{p}_{t-1}\left(\mathbf{x}^{*}\right)\right] \\
= & \overline{\mathbb{E}}\left[p_{t, 1}(1) \mid p_{t-1,1}(1)\right] \\
= & (1-\lambda) p_{t-1,1}(1)+\lambda \overline{\mathbb{E}}\left[r_{t-1,1}^{(s)}(1) \mid p_{t-1,1}(1)\right] \\
= & (1-\lambda) p_{t-1,1}(1)+\lambda \frac{N}{M} p_{t-1,1}(1) \\
= & \left(1+\frac{\lambda(N-M)}{M}\right) p_{t-1,1}(1),
\end{aligned}
$$

where $\overline{\mathbb{E}}[A \mid B]=\mathbb{E}\left[A \mid B, \forall t>0,0 \leq i \leq n: r_{t, i}\left(x_{i}\right)=\right.$ $\left.p_{t-1, i}\left(x_{i}\right)\right]$, and we always use this notation in this paper. According to Assumption 2, we can obtain

$$
\overline{\mathbb{E}}\left[p_{t, 1}(1) \mid \mathbf{p}_{t-1}\left(\mathbf{x}^{*}\right)\right]=\left(1+\frac{\lambda(N-M)}{M}\right)^{t} p_{0,1}(1)
$$

We can set the initial value $p_{0,1}(1)$ to be the initial case $\frac{1}{2}$, then we have,

$$
\overline{\mathbb{E}}\left[p_{t, 1}(1) \mid p_{0,1}(1)\right]=\frac{1}{2}\left(1+\frac{\lambda(N-M)}{M}\right)^{t} .
$$

Now we can solve the equation

$$
\overline{\mathbb{E}}\left[p_{t, 1}(1) \mid p_{0,1}(1)\right]=\frac{M}{N} .
$$

According to Lemma 2, we can achieve the upper case of the convergence time under the average behavior, the expectation of $T_{1}$ will be

$$
\overline{\mathbb{E}}\left[T_{1}\right]<t_{1}+1=\frac{\ln \frac{2 M}{N}}{\ln \left(1+\frac{\lambda(N-M)}{M}\right)}+1
$$

Other bits are with convergence processes similar to the above first bit's case. The value of a bit in an individual is actually influenced by all bits left to it. Let's consider an upper case of the CT, the domino convergence case described in Section 2: all bits converge to the optimum's bits one after another from left to right. (According to Assumption 1, the EDA will converge to the global optimum, hence every bits of the individuals will converge to the optimum's corresponding bits.)

In the upper case of the CT, the marginal probability of every bit has its own period of converging, none of the bits are converging in the same time. In this case, it is easy to find that, once if the $j$-th marginal probability $p_{., j}(1)$ is converging, the $i$-th $(\forall i>j)$ marginal probability $p_{., i}(1)$ will be affected. $p_{., i}(1)$ tends to become more closer to 1 , because letting $i$-th bit of a individual be 1 , rather than 0 , will not cause reducing the fitness, while letting it be 0 , rather than 1 , may cause reducing the average fitness of the population. In other words, IUMDA (UMDA) tends to preserve the values of the bits with the tendencies of increasing the fitness, thus tends to increase $p_{., i}(1)$. So, in expected case, we can assume that at least a value of $\frac{1}{2}$ should be maintained for $p_{., i}(1)$ when the bits left to the $i$-th bits are converging. Thus $p_{\text {.,i }}(1)$ accumulates from $\frac{1}{2}$ to 1 one after another. This upper case can promise the convergence of all bits: for the $i$-th bit, if its left bits have all converged to the optimum's corresponding bits, it will also be forced to converge to the optimum's $i$-th bit, under the selection pressure: on LEADINGONES function, the only way to let the samples' fitness become bigger than $i$ is that we made the $i$-th bit of the solution to be 1 . This trend will be preserved and accumulated by the selection operator. So, similar to (10), (11) and (12), we can achieve the case of the $i$-th bit under the condition that all bits left to the $i$-th bit have converged to 1 ,

$$
\begin{aligned}
& \bar{p}_{t-v_{i-1}, i}(1)=\left(1+\frac{\lambda(N-M)}{M}\right) \bar{p}_{t-v_{i-1}-1, i}(1), \\
& \bar{p}_{v_{i}, i}(1)=\frac{1}{2}\left(1+\frac{\lambda(N-M)}{M}\right)^{t}
\end{aligned}
$$

where $v_{k}=\sum_{j \leq k} T_{k}, k=1, \ldots, n$. Thus we have,

$$
\begin{aligned}
\mathbb{E}[T \mid \forall t & \left.>0,0 \leq i \leq n: r_{t, i}\left(x_{i}\right)=p_{t-1, i}\left(x_{i}\right)\right] \\
& <\sum_{i=1}^{n} \overline{\mathbb{E}}\left[T_{i}\right]<\frac{n \ln \frac{2 M}{N}}{\ln \left(1+\frac{\lambda(N-M)}{M}\right)}+n
\end{aligned}
$$

Corollary 1: For UMDA on LEADINGONES function, its convergence time under the average behavior satisfies:

$$
\begin{aligned}
\mathbb{E}\left[T \mid \forall t>0,0 \leq i \leq n: r_{t, i}\left(x_{i}\right)\right. & \left.=p_{t-1, i}\left(x_{i}\right)\right] \\
< & \frac{n \ln \frac{2 M}{N}}{\ln \left(\frac{N}{M}\right)}+n
\end{aligned}
$$


Corollary 2: For IUMDA on LEADINGONES function, its mean FHT under the average behavior satisfies:

$$
\begin{aligned}
\mathbb{E}[\tau \mid \forall t>0,0 \leq i \leq & \left.n: r_{t, i}\left(x_{i}\right)=p_{t-1, i}\left(x_{i}\right)\right] \\
& <\frac{n \ln \frac{2 M}{N}}{\ln \left(1+\frac{\lambda(N-M)}{M}\right)}+n
\end{aligned}
$$

Proof: This corollary can be easily obtained from Theorem 1 and (5).

\section{FHT of UMDA With TOURNAMENT SELECTION ON LEADINGONES FUNCTION}

Lemma 3: For UMDA and IUMDA with 2-tournament selection, the proportions of the best individuals before and after selection in the $t$-th generation satisfy:

$$
\mathbb{E}\left[R_{t}^{(s)} \mid R_{t}\right]=1-\left(1-R_{t}\right)^{2}
$$

where $R_{t}$ and $R_{t}^{(s)}$ are the proportions of the best individuals before and after 2-tournament selection.

Theorem 2: For UMDA with 2-tournament selection on LEADINGONES function, the convergence time satisfies:

$$
\begin{aligned}
\mathbb{E}\left[T \mid \forall t>0,0 \leq i \leq n: r_{t, i}\left(x_{i}\right)\right. & \left.=p_{t-1, i}\left(x_{i}\right)\right] \\
< & \frac{n \ln N}{2 \ln 2}+n .
\end{aligned}
$$

Proof: As the proof of Theorem 1, For IUMDA, we can estimate the conditional expectation of the first bit's marginal probability distribution $p_{t, 1}(1)$.

$$
\begin{aligned}
& \overline{\mathbb{E}}\left[p_{t, 1}(1) \mid \mathbf{p}_{t-1}\left(\mathbf{x}^{*}\right)\right] \\
= & \overline{\mathbb{E}}\left[p_{t, 1}(1) \mid p_{t-1,1}(1)\right] \\
= & \overline{\mathbb{E}}\left[p_{t-1,1}(1)+\lambda\left(r_{t-1,1}^{(s)}(1)-p_{t-1,1}(1)\right) \mid p_{t-1,1}(1)\right] \\
= & (1-\lambda) p_{t-1,1}(1)+\lambda \overline{\mathbb{E}}\left[r_{t-1,1}^{(s)}(1) \mid p_{t-1,1}(1)\right] \\
= & (1-\lambda) p_{t-1,1}(1)+\lambda\left(1-\left(1-p_{t-1,1}(1)\right)^{2}\right) \\
= & (\lambda+1) p_{t-1,1}(1)-\lambda p_{t-1,1}^{2}(1)
\end{aligned}
$$

For the case of UMDA, the control parameter $\lambda=1$ in the above equations. Thus we have,

$$
\overline{\mathbb{E}}\left[p_{t, 1}(1) \mid p_{t-1,1}(1)\right]=2 p_{t-1,1}(1)-p_{t-1,1}^{2}(1)
$$

The above equation is equivalent to the following one:

$$
\overline{\mathbb{E}}\left[p_{t, 1}(1) \mid p_{t-1,1}(1)\right]-1=-\left(p_{t-1,1}(1)-1\right)^{2} .
$$

Approximating by the average behavior of 2-tournament selection, we have:

$$
\overline{\mathbb{E}}\left[p_{t, 1}(1) \mid p_{0,1}(1)\right] \approx 1-\left(p_{0,1}(1)-1\right)^{2 t} .
$$

In order to get the $\epsilon$-convergence time, we have,

$$
\overline{\mathbb{E}}\left[p_{t_{1}, 1}(1) \mid p_{0,1}(1)\right]=1-\epsilon .
$$

We set the initial value of $p_{0,1}(1)$ to $\frac{1}{2}$, finally we can get,

$$
t_{1} \approx \frac{\ln \epsilon}{-2 \ln 2} .
$$

We set $\epsilon<\frac{1}{N}$. We also know that $\overline{\mathbb{E}}\left[p_{t, 1}(1) \mid p_{0,1}(1)\right]=1-\epsilon$, thus in expectation, the UMDA with tournament selection can make the first bit converge to 1 with only one more generation,

$$
\begin{gathered}
\overline{\mathbb{E}}\left[p_{t_{1}+1, i}(1) \mid p_{t_{1}, i}(1)=1-\epsilon, \epsilon<\frac{1}{N}\right] \\
=\overline{\mathbb{E}}\left[r_{t_{1}, i}^{(s)}(1) \mid p_{t_{1}, i}(1)>1-\frac{1}{N}\right]=1 .
\end{gathered}
$$

Hence we can get,

$$
\overline{\mathbb{E}}\left[T_{1}\right]<t_{1}+1 \approx \frac{\ln N}{2 \ln 2}+1 .
$$

To the other bits, similar processes can together build the upper case of UMDA as we have done in the proof of Theorem 1 for IUMDA:

$$
\begin{aligned}
\mathbb{E}[T \mid \forall t>0,0 \leq i & \left.\leq n: r_{t, i}\left(x_{i}\right)=p_{t-1, i}\left(x_{i}\right)\right] \\
& <\sum_{i=1}^{n} \overline{\mathbb{E}}\left[T_{i}\right]<\frac{n \ln N}{2 \ln 2}+n .
\end{aligned}
$$

Thus we complete the proof of Theorem 2.

Corollary 3: For UMDA on LEADINGONES function, its mean FHT under the average behavior satisfies:

$$
\begin{aligned}
\mathbb{E}\left[\tau \mid \forall t>0,0 \leq i \leq n: r_{t, i}\left(x_{i}\right)\right. & \left.=p_{t-1, i}\left(x_{i}\right)\right] \\
& <\frac{n \ln N}{2 \ln 2}+n .
\end{aligned}
$$

Proof: This corollary can easily be obtained by Theorem 2 and (5).

As we discussed above, LEADINGONES function is actually an easy problem for IUMDA or UMDA. The main reason is that LEADINGONES function is a unimodal function, and the building blocks with high fitness can always be preserved, and their lengths increase constantly. Finally, the global optima is built. However, this phenomena does not happen in the cases of some deceptive problems.

\section{FHT OF IUMDA WiTh TOURNAMENT SELECTION ON TRAPLEADINGONES FUNCTION}

\section{A. Related Discussions}

He and Yao [21] divided the optimization problems into two class according to the mean FHTs of EAs:

Easy Class: For a given EA, starting from any intial population, the mean FHT needed by EA to reach the optimum is polynomial in the problem size.

Hard Class: For a given EA, starting from any intial population, the mean FHT needed by EA to reach the optimum is exponential in the problem size.

In other words, the hardness of a problem to a given EA can be measured by the mean FHT of the EA. As a special kind of EAs, this classification is also suitable for EDAs.

In this paper, the upper bounds of the mean FHTs of IUMDA and UMDA with truncation and tournament selection strategies on LEADINGONES function have been obtained, which shows that the IUMDA and UMDA can find the global optimum of LEADINGONES function within 
$O(n)$ generations under Assumption 1. Hence it is reasonable for us to believe that LEADINGONES function is "easy" for IUMDA and UMDA as it is "easy" for GAs [10], [11], [?] according to He and Yao's classification in [21].

\section{B. A Hard Problem for IUMDA}

A natural idea is that, we should also study some "hard" problems. As a case study, we are trying to find such a deceptive problem for IUMDA and prove that it is "hard" for UMDA with the increasing problem size $n$. Let us consider the following maximization problem called TRAPLEADINGONES:

$$
\text { TrapleadingOnes }(\mathbf{x})= \begin{cases}g(\mathbf{x}), & g(\mathbf{x}) \leq n, \\ -n, & g(\mathbf{x})>n,\end{cases}
$$

where $g(\mathbf{x})=n x_{n}+\sum_{i=1}^{n-1} \prod_{j=1}^{i} x_{j}, x_{j}=0$ or $1(j=$ $1, \ldots, n)$. The global optimum of TRAPLEADINGONES function is $\mathbf{x}^{*}=(0, \ldots, 0,1)$. This function is similar as the instance of the Subset Sum Problem studied in [10] and [15]. It belongs to the wide-gap problem [22]. It is easy for us to prove that the problem is "hard" for GAs by drift analysis [10], [13] or by Yu and Zhou 's approach [15].

How about the IUMDA and UMDA's cases? On this issue, still under Assumption 1, we have the following lemma,

Lemma 4: For IUMDA with 2-tournament selection on TRAPLEADINGONES function, $\forall i=1, \ldots, n$, we have,

$$
\overline{\mathbb{E}}\left[p_{t, i}\left(x_{i}^{*}\right) \mid \mathbf{p}_{t-1}\left(\mathbf{x}^{*}\right)\right] \leq p_{0, i}\left(x_{i}^{*}\right)=\frac{1}{2},
$$

where $\left(x_{1}^{*}, \ldots, x_{n-1}^{*}, x_{n}^{*}\right)=\mathbf{x}^{*}=(0, \ldots, 0,1)$.

$$
\text { Proof: }
$$

First, let us study the situation of the value of the $n$-th bit $x_{n}$ of a given individual $\mathbf{x}$. It is obvious that if $x_{n}=1$ and one or more rest bits take the value of 0 , the fitness of the individual will drop to a very low level. In this case, the IUMDA tends to select those individuals with its $n$-th bits to be 0 . We can estimate the conditional expectation of $r_{t, n}^{(s)}\left(x_{i}^{*}\right)$ by the property of the 2-tournament selection:

$$
\begin{aligned}
& \overline{\mathbb{E}}\left[r_{t, n}^{(s)}\left(x_{i}^{*}\right) \mid \mathbf{p}_{t-1}\left(\mathbf{x}^{*}\right)\right] \\
= & p_{t-1, n}^{2}(1)\left(1-\prod_{i=1}^{n-1} p_{t-1, i}(0)\right) \\
& +\left(1-\left(1-p_{t-1, n}(1)\right)^{2}\right) \prod_{i=1}^{n-1} p_{t-1, i}(0),
\end{aligned}
$$

and we can then have

$$
\begin{aligned}
& \overline{\mathbb{E}}\left[p_{t, n}\left(x_{n}^{*}\right)-p_{t-1, n}\left(x_{n}^{*}\right) \mid \mathbf{p}_{t-1}\left(\mathbf{x}^{*}\right)\right] \\
= & \overline{\mathbb{E}}\left[\lambda\left(r_{t-1, n}^{(s)}(1)-p_{t-1, n}(1)\right) \mid \mathbf{p}_{t-1}\left(\mathbf{x}^{*}\right)\right] \\
= & -\lambda p_{t-1, n}(1)+\lambda p_{t-1, n}^{2}(1)\left(1-\prod_{i=1}^{n-1} p_{t-1, i}(0)\right) \\
& +\lambda\left(1-\left(1-p_{t-1, n}(1)\right)^{2}\right) \prod_{i=1}^{n-1} p_{t-1, i}(0) .
\end{aligned}
$$

Assume that $t=1$, then $\prod_{i=1}^{n-1} p_{0, i}(0)=1 / 2^{n-1}$ is very small, hence we can omit it. Approximately, we have:

$$
\begin{aligned}
& \overline{\mathbb{E}}\left[p_{1, n}\left(x_{n}^{*}\right)-p_{0, n}\left(x_{n}^{*}\right) \mid \mathbf{p}_{0}\left(\mathbf{x}^{*}\right)\right] \\
\approx & -\lambda p_{0, n}(1)+\lambda p_{0, n}^{2}(1)<0 .
\end{aligned}
$$

For the marginal probability of the first bit, we have,

$$
\begin{aligned}
& \overline{\mathbb{E}}\left[p_{t, 1}\left(x_{1}^{*}\right)-p_{t-1,1}\left(x_{1}^{*}\right) \mid \mathbf{p}_{t-1}\left(\mathbf{x}^{*}\right)\right] \\
= & -\overline{\mathbb{E}}\left[\lambda\left(r_{t-1,1}^{(s)}(1)-p_{t-1,1}(1)\right) \mid \mathbf{p}_{t-1}\left(\mathbf{x}^{*}\right)\right] \\
< & \left(p_{t-1, n}(1)-1\right)\left(\lambda p_{t-1,1}(1)-\lambda p_{t-1,1}^{2}(1)\right)<0
\end{aligned}
$$

At the same time, if the $n$-th bit of an individual is 0 , and the $j$-th marginal probability $p_{., j}(1)$ is becoming large, the $i$-th $(\forall n>i>j)$ marginal probability $p_{., i}(1)$ will be affected. In this case, it is obvious that $p_{., i}(1)$ tends to become more closer to 1 , because letting $i$-th bit of a individual be 1 , rather than 0 , will not cause reducing the fitness, while letting it be 0 , rather than 1 , may cause reducing the average fitness of the population. If the $n$-th bit of a non-optimal individual is 1 , its fitness is $-n$, and it will not be affected by other bits. In other words, IUMDA (UMDA) tends to preserve the values of the bits with the tendencies of increasing the fitness, thus tends to increase $p_{., i}(1)$. So, in expected case, we can assume that at least a value of $\frac{1}{2}$ should be maintained for $p_{., i}(1)$ when the bits left to the $i$-th bits are becoming larger.

Now it is easy to generalize (20) to the $j$-th bit's case. We can find that $p_{t, i}\left(x_{i}^{*}\right)$ (where $i=1, \ldots, n-1$ ) is reducing by similar analysis:

$$
\begin{aligned}
& \overline{\mathbb{E}}\left[p_{t, i}\left(x_{i}^{*}\right)-p_{t-1, i}\left(x_{i}^{*}\right) \mid \mathbf{p}_{t-1}\left(\mathbf{x}^{*}\right)\right] \\
= & -\overline{\mathbb{E}}\left[\lambda\left(r_{t-1, i}^{(s)}(1)-p_{t-1, i}(1)\right) \mid \mathbf{p}_{t-1}\left(\mathbf{x}^{*}\right)\right] \\
< & \left(p_{t-1, n}(1)-1\right)\left(\lambda p_{t-1, i}(1)-\lambda p_{t-1, i}^{2}(1)\right)<0
\end{aligned}
$$

So, $\prod_{i=1}^{n-1} p_{t, i}(0)$ tends to become smaller and smaller, so similar approximations as (19) are always acceptable when $t$ become larger. Thus $\bar{p}_{t, i}\left(x_{i}^{*}\right)(\forall i=1, \ldots, n)$ tends to become smaller when $t$ become larger. We have,

$$
\overline{\mathbb{E}}\left[p_{t, i}\left(x_{i}^{*}\right) \mid \mathbf{p}_{t-1}\left(\mathbf{x}^{*}\right)\right]<\frac{1}{2} .
$$

Now we can reach the following theorem:

Theorem 3: For IUMDA with 2-tournament selection on TRAPLEADINGONES function, if it will converge to the global optimum, then its corresponding mean FHT satisfies

$$
\begin{aligned}
\mathbb{E}\left[\tau \mid \forall t>0,0 \leq i \leq n: r_{t, i}\left(x_{i}\right)=\right. & \left.p_{t-1, i}\left(x_{i}\right)\right] \\
& >\frac{1}{N}\left(\frac{4}{3}\right)^{n} .
\end{aligned}
$$

Proof:

We know that the 2-tournament selection is a random operator, and its expected behavior is analyzed in the last section. But in order to carry out a more precise analysis, here we take into account its random performance. We allow the selection operator performs its random behavior in every generation, but the history information before this generation 
still uses the expected case, which reduces the complexity of analyzing the random sequence. We know the population size $M$ is very large, but have not reach an infinite size, then according to De Moivre-Laplace central limit theorem in the probability theory, approximately, we have

$$
\forall i, t: \quad \frac{r_{t, i}^{(s)}\left(x_{i}^{*}\right)-\bar{r}_{t, i}^{(s)}\left(x_{i}^{*}\right)}{\sqrt{\frac{\bar{r}_{t, i}^{(s)}\left(x_{i}^{*}\right)\left(1-\bar{r}_{t, i}^{(s)}\left(x_{i}^{*}\right)\right)}{M}}} \sim N(0,1)
$$

where $\bar{r}_{t, i}^{(s)}\left(x_{i}^{*}\right)=\overline{\mathbb{E}}\left[r_{t, n}^{(s)}\left(x_{i}^{*}\right) \mid \mathbf{p}_{t-1}\left(\mathbf{x}^{*}\right)\right], N(0,1)$ is the standard Gaussian distribution. Thus in the $t$-th generation, for $\epsilon_{i, t}>0$, we have,

$$
\begin{array}{r}
\mathbb{P}\left(r_{t, i}^{(s)}\left(x_{i}^{*}\right)<\bar{r}_{t, i}^{(s)}\left(x_{i}^{*}\right)+\epsilon_{i, t}\right) \\
=\Phi\left(\epsilon_{i, t} / \sqrt{\frac{\bar{r}_{t, i}^{(s)}\left(x_{i}^{*}\right)\left(1-\bar{r}_{t, i}^{(s)}\left(x_{i}^{*}\right)\right)}{M}}\right),
\end{array}
$$

where $\Phi($.$) is the probability distribution of N(0,1) . \forall t>$ $0, i=1,2,3, n-1$, let $\epsilon_{i, t}=\sqrt{\bar{r}_{t, i}^{(s)}\left(x_{i}^{*}\right)\left(1-\bar{r}_{t, i}^{(s)}\left(x_{i}^{*}\right)\right)}$, according to (21) and Lemma 4 , we have

$$
0<\epsilon_{i, t} \leq \frac{1}{2} \sqrt{\bar{r}_{1, i}^{(s)}\left(x_{i}^{*}\right)\left(1-\bar{r}_{1, i}^{(s)}\left(x_{i}^{*}\right)\right)}=\epsilon<\frac{1}{4},
$$

where $\epsilon$ is a constant. We know that $r_{t, i}^{(s)}\left(x_{i}^{*}\right)<\bar{r}_{t, i}^{(s)}\left(x_{i}^{*}\right)+\epsilon$ holds with the probability of $\Phi(\sqrt{M} / 2)$. Since $M$ is very large, we can omit the extremely small probability of the event $r_{t, i}^{(s)}\left(x_{i}^{*}\right)>\bar{r}_{t, i}^{(s)}\left(x_{i}^{*}\right)+\epsilon$. Hence the below inequality holds approximately under our assumption at the beginning of this proof:

$$
\begin{aligned}
& p_{t, i}\left(x_{i}^{*}\right) \\
= & \bar{p}_{t-1,1}\left(x_{i}^{*}\right)+\lambda\left(r_{t-1, i}^{(s)}\left(x_{i}^{*}\right)-\bar{p}_{t-1, i}\left(x_{i}^{*}\right)\right) \\
< & \bar{p}_{t-1,1}\left(x_{i}^{*}\right)+\lambda\left(\bar{r}_{t-1, i}^{(s)}\left(x_{i}^{*}\right)+\epsilon-\bar{p}_{t-1, i}\left(x_{i}^{*}\right)\right) \\
< & \bar{p}_{t, 1}\left(x_{i}^{*}\right)+\lambda \epsilon<\frac{1}{2}+\lambda \epsilon .
\end{aligned}
$$

(23) is obtained by Lemma 4. Then we have

$$
p_{t}\left(\mathbf{x}^{*}\right)=\prod_{i=1}^{n} p_{t, i}\left(x_{i}^{*}\right)<\left(\frac{1}{2}+\lambda \epsilon\right)^{n} .
$$

At the end of the $t$-th generation, IUMDA will sample $N$ individuals based on the probability $p_{t}(\mathbf{x})$. The probability that one or more individuals of the sample hit the global optimum is

$$
\begin{aligned}
& \mathbb{P}\left(\mathbf{x}^{*} \in \xi_{t} \mid \mathbf{x}^{*} \notin \xi_{t-1}\right) \\
= & \sum_{Y \in E} \mathbb{P}\left(\mathbf{x}^{*} \in \xi_{t} \mid \xi_{t-1}=Y\right) \mathbb{P}\left(\xi_{t-1}=Y\right) \\
< & \left(1-\left(1-\left(\frac{1}{2}+\lambda \epsilon\right)^{n}\right)^{N}\right) \sum_{Y \in E} \mathbb{P}\left(\xi_{t-1}=Y\right) \\
< & 1-\left(1-\left(\frac{1}{2}+\lambda \epsilon\right)^{n}\right)^{N} \sim N\left(\frac{1}{2}+\lambda \epsilon\right)^{n},
\end{aligned}
$$

where $E$ is the population set. All elements of $E$ doesn't contain the global optimum. Since $N$ is very large, (25) is obtained by approximation. Suppose $G$ is a random variable, and $G$ obeys the geometric distribution, with the parameter $N\left(\frac{1}{2}+\epsilon\right)^{n}$, then we have,

$$
\mathbb{E}[G]=\frac{1}{N}\left(\frac{2}{1+2 \lambda \epsilon}\right)^{n}
$$

Moreover, according to (25), for the FHT $\tau$ of IUMDA, we have

$$
\begin{aligned}
& \mathbb{P}\left(\tau \leq k \mid \forall t>0,0 \leq i \leq n: r_{t, i}\left(x_{i}\right)=p_{t-1, i}\left(x_{i}\right)\right) \\
= & 1-\prod_{t=1}^{k}\left(1-\mathbb{P}\left(\mathbf{x}^{*} \in \xi_{t} \mid \mathbf{x}^{*} \notin \xi_{t-1}\right)\right) \\
< & 1-\left(1-N\left(\frac{1}{2}+\lambda \epsilon\right)^{n}\right)^{k}=\mathbb{P}(G \leq k) .
\end{aligned}
$$

Notice that $0<\lambda \leq 1$ and $\epsilon<\frac{1}{4}$, according to (26) and Lemma 2 in [15], we can obtain:

$$
\begin{aligned}
\mathbb{E}[\tau \mid \forall t & \left.>0,0 \leq i \leq n: r_{t, i}\left(x_{i}\right)=p_{t-1, i}\left(x_{i}\right)\right] \\
>\mathbb{E}[G] & =\frac{1}{N}\left(\frac{2}{1+2 \lambda \epsilon}\right)^{n}>\frac{1}{N}\left(\frac{4}{3}\right)^{n} .
\end{aligned}
$$

Now we have shown that TRAPLEADINGONES function is bounded below by an exponential function of the problem size $n$, hence it is a "hard" problem for IUMDA. It is clear that if the population size $N$ increases, the lower bound will decrease; However, if $N$ is also an exponential function of $n$, this bound may become trivial.

\section{CONCLUDING REMARKS}

We have obtained the upper bounds of the mean CTs of UMDA and IUMDA on LEADINGONES function. The upper bounds are all linear function of the problem size if we omit the relation between population sizes and problem size. According to (2) and (5), we know that the mean FHTs are always no longer than the expected CTs, thus the mean FHTs are also with the same upper bound with the mean CTs, which is also a linear function of the problem size. This result fits the experiment result about UMDA on LEADINGONES function in [8] very well. We have also studied a "hard" problem for the IUMDA. It is shown that the mean FHT of IUMDA on TRAPLEADINGONES function is exponentially large. It is the first time that the mean FHTs of UMDA and IUMDA, including both polynomial and exponential case, are estimated rigorously. However, it is noteworthy that we assume a very large population size in our analysis, which is different from the real situation. In the real situation, the performance of EDAs varies near the expected case in every generation; If it is further combined with relatively small population size, the time complexity results may be different.

The UMDA and IUMDA are in a very simple form, they don't consider the dependencies among variables. Due to the phenomenon of domino convergence, they perform very well on the LEADINGONES function, whose variables are dependent with one another, and the dependencies among 
the bits of the solution are varying in different stages of IUMDA. But on the TRAPLEADINGONES function, the fitness structure mislead the search of IUMDA. Our future work includes the study of more complex EDAs on simple or complex problems and the study of the relationship between the models of EDAs and the problem hardness.

\section{ACKNOWLEDGMENT}

This work is partially supported by two National Natural Science Foundation of China grants (No. 60428202 and 60533020) and an Engineering and Physical Science Research Council grant in UK (No. EP/C520696/1).

\section{REFERENCES}

[1] H. Mühlenbein and G. Paaß, "From recombination of genes to the estimation of distribution i. binary parameters." In Lecture Notes in Computer Science 1411: Parallel Problem Solving from Nature - PPSN IV: 178-187, 1996

[2] H. Mühlenbein and D. Schlierkamp-Voosen, "Predictive models for the breeder genetic algorithm, I.: continuous parameter optimization." Evolutionary Computation 1(1): 25-49, 1993.

[3] H. Mühlenbein, "The equation for response to selection and its use for prediction." Evolutionary Computation 5(3): 303-346, 1997.

[4] M. Pelikan, K. Sastry and D. E. Goldberg, "Evolutionary Algorithms + Graphical Models = Scalable Black-Box Optimization." Illinois Genetic Algorithms Labortaory, University of Illinois ar UrbanaChampaign, IlliGAL Rep. 2001029, 2001.

[5] S. Droste, "A Rigorous Analysis of the Compact Genetic Algorithm for Linear Functions." Natural Computing, 5(3): 257-283, 2006

[6] G. R. Harik, F. G. Lobo and D. E. Goldberg, "The compact gentetic algorithm." In Proceedings of the 1998 IEEE International Conference on Evolutionary Computation: 523-528, 1998.

[7] R. Rastegar and M. R. Meybodi, "A study on global convergence time complexity of estimation of distribution algorithms." In Lecture Notes in Artificial Intelligence 3641: Rough Sets, Fuzzy Sets, Data Mining and Granular Computing (RSFDGrC2005): 441-450, 2005.

[8] C. González, A. Ramírez, J. A. Lozano, P. Larraãga, "Average Time Complexity of Estimation of Distribution Algorithms." In Lecture Notes in Computer Science 3512: the 8th International WorkConference on Artificial Neural Networks (IWANN'2005): 42-49, 2005.
[9] G. Rudolph, "Finite Markov chain results in evolutionary computation: A tour d'horizon," Fundamenta Informaticae, 35(1-4) 67-89, 1998.

[10] J. He and X. Yao, "Drift analysis and average time complexity of evolutionary algorithms," Artificial Intelligence, 127(1): 57-85, 2001.

[11] S. Droste, T. Jansen, and I. Wegener, "On the analysis of the $(1+1)$ evolutionary algorithm." Theoretical Computer Science 276(1-2): 51$81,2002$.

[12] J. Horn, D. E. Goldberg and K. Deb, "Long path problems." In Lecture Notes in Computer Science 886: Parallel Problem Solving from Nature - PPSN III: 149-158, 1994.

[13] L. Ding and J. Yu, Some theoretical results about the computation time of evolutionary algorithms In Proceedings of the Genetic and Evolutionary Computation Conference (GECCO2005): 1409-1415, 2005.

[14] J. He and X. Yao, "Towards an Analytic Framework for Analysing the Computation Time of Evolutionary Algorithms," Artificial Intelligence, 145(1-2): 59-97, 2003.

[15] Y. Yu and Z.-H. Zhou, "A new approach to estimating the expected first hitting time of evolutionary algorithms." In Proceedings of the 21st National Conference on Artificial Intelligence (AAAI'06): 555560, 2006.

[16] M. Pelikan, K. Sastry and D. E. Goldberg, "Scalability of the bayesian optimization algorthm." International Journal of Approximate Reasoning, 31: 221-258, 2002.

[17] H. Mühlenbein and T. Mahnig, "Evolutionary optimization and the estimation of search distrubutions with applications to graph bipartitioning." International Journal of Approximate Reasoning, 31: 157192, 2002.

[18] Q. Zhang and H. Mühlenbein, "On the convergence of a class of estimation of distribution algoirthms." IEEE Transations on Evolutionary Computation, 8(2):127-136, 2004

[19] M. Rudnick, "Genetic algorithms and fitness variance with an application to the automated design of artificial neural networks." Unpublished doctoral dissertation, Oregon Graduate Institue of Science and technology.

[20] D. Thierens, D. E. Goldberg and A.G. Pereira, "Domino convergence, drift, and the temporal-salience structure ofproblems." In Proceedings of the 1998 IEEE International Conference on Evolutionary Computation: 535-540, 1998.

[21] J. He and X. Yao, "A study of drift analysis for estimating computation time of evolutionary algorithms," Natural Computing (3): 21-35, 2004.

[22] J. He, C. Reeves and X. Yao, "A Discussion on posterior and prior measures of problem diffciulties." (accepted by PPSN IX Workshop on Evolutionary Algorithms - Bridging Theory and Practice), 2006. 